\title{
DJ-1 knockdown inhibits growth and xenograft-induced tumor generation of human hepatocellular carcinoma cells
}

\author{
SHUNFANG LIU ${ }^{1}$, GUOXIAN LONG ${ }^{1}$, HONGLAN WEI $^{2}$, LEI SHI ${ }^{1}$, ZHIFANG YANG $^{3}$, \\ DONGBO LIU ${ }^{1}$, GUOQING HU ${ }^{1}$ and HONG QIU ${ }^{1}$ \\ ${ }^{1}$ Department of Oncology, Tongji Hospital of Tongji Medical College, Huazhong University of Science and Technology, \\ Wuhan, Hubei 430030; ${ }^{2}$ Department of Nephrology, Puai Hospital of Tongji Medical College, Huazhong University \\ of Science and Technology, Wuhan, Hubei 430033; ${ }^{3}$ Department of General Surgery, Tongji Hospital of Tongji \\ Medical College, Huazhong University of Science and Technology, Wuhan, Hubei 430030, P.R. China
}

Received August 13, 2014; Accepted October 17, 2014

DOI: 10.3892/or.2014.3594

\begin{abstract}
The aim of this study was to identify potential downstream effectors of the oncogene DJ-1 in hepatocellular carcinoma (HCC) cell lines, and examine its role in the Akt signaling pathway and HCC oncogenesis. Expression of the DJ-1 protein was assessed by immunoblotting in several human HCC cell lines. Knockdown of DJ-1 was achieved by transfecting DJ-1-specific short hairpin RNAs into the HepG2 HCC cell line. The effect of DJ-1 downregulation on phosphatase and tensin homolog (PTEN) and phosphorylated Akt was evaluated. In addition, cell cycle, proliferation, adhesion and invasion were analyzed in the DJ-1 knockdown of HepG2 cells. The growth of HepG2-induced tumor was evaluated in a nude mouse model after DJ-1 silencing. Stable DJ-1 knockdown was achieved in HepG2 cells using a shRNA eukaryotic expression vector. Downregulation of DJ-1 increased PTEN expression but decreased phosphorylation of Akt in HepG2 cells. In addition, DJ-1 knockdown resulted in the decreased proliferation, adhesion and invasion of HepG2 cells in vitro, and inhibited the growth of HepG2-induced tumor in vivo. DJ-1 knockdown altered the malignant behavior of HepG2 cells, potentially through the Akt signaling pathway, suggesting a crucial role for DJ-1 in the oncogenesis of HCC.
\end{abstract}

\section{Introduction}

Hepatocellular carcinoma (HCC) is one of the most common types of cancer worldwide and causes approximately one million deaths each year (1-3). The incidence of HCC in the USA has increased in the past two decades, possibly due to

Correspondence to: Dr Hong Qiu, Department of Oncology, Tongji Hospital of Tongji Medical College, Huazhong University of Science and Technology, 1095 Jie Fang Avenue, Wuhan, Hubei 430030, P.R. China

E-mail: qiuhongmedsci@163.com

Key words: HCC, DJ-1, HepG2, Akt signaling pathways increased incidence in cirrhosis and longstanding hepatitis $\mathrm{C}$ infection (1). HCC is considered a major health problem due to endemic hepatitis $\mathrm{B}$ and $\mathrm{C}$ and regional exposure to environmental pathogens (3). HCC is invasive and resistance to chemotherapeutic agents limits treatment options. At present, the only curative treatment is surgery (4). Surgical resection offers the best prognosis when the tumor is $<5 \mathrm{~cm}$ in diameter and limited to one lobe of the liver, has not invaded the liver vasculature, and liver function is well preserved. However, long-term survival is rare, mainly due to the recurrence and metastasis of HCC (5). Identification of target genes associated with the progression of $\mathrm{HCC}$ and improved understanding of the early molecular events in tumorigenesis may lead to improvements in diagnostic efficiency and aid in the development of new therapeutic strategies for HCC.

Apoptosis is a genetically determined process of controlled cellular suicide (6). Deregulation of apoptosis is involved in the pathophysiology of liver disease including hepatocarcinogenesis (7). HCC cell resistance to apoptosis impairs the efficacy of HCC therapeutic regimens (8). The oncogene DJ-1 encodes a ubiquitously expressed 189 -amino acid integral mitochondrial protein reported to confer resistance to apoptosis (9-13). DJ-1 has been reported to regulate cell death, and appears to play a role in tumorigenesis, invasion and metastasis in breast cancer, HCC, non-small cell lung carcinoma and prostate cancer (9-17). The oncogenic effect of DJ-1 is mainly attributed to its antiapoptotic ability. A number of DJ-1 downstream effectors, such as PTEN, Akt, IKK and NF- $\kappa \mathrm{B}$, are involved in regulating the progression and invasion of tumors $(16,18)$.

In the present study, we aimed to investigate the role of DJ-1 in HCC tumorigenesis. HepG2 cells provide an in vitro model system for the study of polarized human hepatocytes, exhibiting robust morphological and functional differentiation with apical and basolateral cell surface domains. Selective shRNA expression vector-mediated RNAi is an effective method for suppressing oncogene products in vitro (19). Therefore, shRNA can be therapeutically used for the inhibition of aberrant mRNA expression in cancer cells (20).

Using a shRNA expression vector, stable DJ-1 knockdown of HepG 2 cells was obtained. We found that DJ-1 downregulation resulted in the growth inhibition of HepG2 cells in vitro 
and in vivo. Increased PTEN expression and decreased Akt phosphorylation were detected in DJ-1 knockdown HepG2 cells.

These findings suggested that DJ-1 knockdown altered the malignant behavior of HepG2 cells, potentially through Akt signaling, indicating a crucial role for DJ-1 in the oncogenesis of HCC.

\section{Materials and methods}

Cell culture. The human HepG2, SMMC-7721 and Huh-7 HCC cell lines were obtained from the China Center for Type Culture Collection (Wuhan University, Wuhan, China). Human metastatic MHCC-97L and MHCC-97H HCC cell lines were obtained from the Liver Cancer Institute of Zhongshan Hospital (Fudan University, Shanghai, China) (21). Cells were cultured at $37^{\circ} \mathrm{C}$ with $5 \% \mathrm{CO}_{2}$ in Dulbecco's modified Eagle's medium (DMEM) high-glucose medium containing 10\% calf serum (both from Gibco-Life Technologies, Carlsbad, CA, USA) and $1 \%$ penicillin and streptomycin.

Construction of DJ-1 shRNA expression plasmid. A candidate DJ-1 cDNA sequence (GenBank NM_007262: AGTGTAGCC GTGATGTGGT) was selected for RNA interference (RNAi). The oligonucleotide sequence used for DJ-1 silencing was BamHII + sense + loop + antisense + terminator + SalI + HindII, with shRNA-DJ-1 (forward), 5'-CACCAGTGTAGC CGTGATGTGGTTTCAAGACGACCACATCACGGCT ACACTTTTTTTG-3' and (reverse), 5'-AGCTCAAAAAAA GTGTAGCCGTGATGTGGTCGTCTTGAAACCACATCA CGGCTACACT-3'. The negative control sequences were: shRNA-HK (forward), 5'-CACCTTTTTTCAAGACGGATG AACTTCAGGGTCAGCTTTTTTG-3' and (reverse), 5'-AGC TCAAAAAAGCTGACCCTGAAGTTCATCCGTCTTGA AAAAA-3'. The underlined sequences denote the loop. An oligonucleotide targeting GAPDH served as a positive control while an irrelevant oligonucleotide served as a negative control. Recombinant plasmid synthesis and purification was performed by Wuhan Genesil Biotechnology Co., Ltd. (Wuhan, China).

Stable transfection of HepG2 with recombinant human DJ-1 shRNA plasmid. HepG2 cells at $60-80 \%$ confluency were transfected with constructed plasmids using Lipofectamine 2000 (Life Technologies, Carlsbad, CA, USA) according to the manufacturer's instructions. At $48 \mathrm{~h}$ after transfection $450 \mu \mathrm{g} / \mathrm{ml} \mathrm{G} 418$ was added to the cell culture medium. The cells were cultured in medium containing G418 for 8 weeks to select stable transfectants. Individual clones were isolated and expanded. The green fluorescence expressed by stable transfectant cells was observed by fluorescence microscopy.

RNA extraction and semi-quantitative PCR. Total RNA was isolated using the TRIzol reagent (Invitrogen-Life Technologies, Carlsbad, CA, USA) according to the manufacturer's instructions, $48-72 \mathrm{~h}$ after transfection. Total RNA $(2 \mu \mathrm{g})$ was used for semi-quantitative reverse transcription PCR on a Bio-Rad S1000 Thermal Cycler (Bio-Rad, Hercules, CA, USA) (Promega, Madison, WI, USA). The primers used were: DJ-1, 5'-AGCAGAGGAAATGGAGACG-3' (forward), and 5'-GCCAACAGAGCAGTAGGAC-3' (reverse); internal control $\beta$-actin 5'-TCTACAATGAGCTGCGTGTG-3' (forward), and 5'-ATCTCCTTCTGCATCCTGTC-3' (reverse). PCR products $(5 \mu \mathrm{l})$ were analyzed by electrophoresis on $1.2 \%$ agarose gels.

Immunoblotting. Cells were collected, washed with phosphate-buffered saline (PBS) three times, and lysed in cell lysis buffer containing RIPA (ProMab, Changsha, China) and protease inhibitor (Promega). The cell lysates were submitted to centrifugation at $12,000 \mathrm{x}$ g for $30 \mathrm{~min}$ and the supernatants were collected as total protein, and quantified by the bicinchoninic acid (BCA) method. Protein $(50 \mu \mathrm{g})$ was used for electrophoresis followed by transfer on PVDF membranes, and probed with rabbit polyclonal antibodies against human DJ-1 (Proteintech, China), PTEN (Epitomics, Inc., Burlingame, CA, USA) or rabbit monoclonal antibodies against human $\beta$-actin (Abzoom Biolabs, Dallas, TX, USA). Following incubation with horseradish peroxidase-conjugated anti-rabbit secondary antibodies, signals were detected with an enhanced chemiluminescence (ECL) chromogenic substrate. Visualization and quantification were carried out on a digital image analyzer.

Flow cytometric analysis. A single-cell suspension of HepG2 cells in the logarithmic phase was seeded in new culture flasks and incubated for $48 \mathrm{~h}$ followed by fixation with $75 \%$ ethanol at $4^{\circ} \mathrm{C}$ overnight. The washed cells were resuspended in PBS containing RNase $\mathrm{A}$, and incubated at $37^{\circ} \mathrm{C}$ for $30 \mathrm{~min}$ prior to the addition of $50 \mu \mathrm{g} / \mathrm{ml}$ propidium iodide (PI) for $30 \mathrm{~min}$ in the dark. After washing, the cells were analyzed using a flow cytometer (Chemunex, Vernon Hills, IL, USA).

Cell growth assay. Cell proliferation was assessed by a colorimetric 3-(4,5-dimethylthiazol-2-yl)-2,5-diphenyltetrazolium bromide (MTT) assay. The cells in the logarithmic phase were seeded at a density of $1 \times 10^{2} / \mu 1$ in triplicate in 96 -well plates (100 $\mu \mathrm{l} /$ well). After 24, 48, 79 and $96 \mathrm{~h}, 50 \mu \mathrm{l}$ MTT $(5 \mathrm{mg} / \mathrm{ml})$ were added to each well and incubated at $37^{\circ} \mathrm{C}$. After $4 \mathrm{~h}$, the supernatants were carefully aspirated and the purple crystals dissolved with $150 \mu \mathrm{l}$ DMSO. Absorbance was measured at $570 \mathrm{~nm}$ on a microtiter plate reader.

Adhesion and invasion assays. Cellular adhesion to plastic and laminin- or fibronectin-coated surfaces was evaluated in 96-well plates. The plates were coated with $20 \mu 1$ DMEM containing $2 \% \mathrm{BSA}$ at $37^{\circ} \mathrm{C}$ for $1 \mathrm{~h}$ and rinsed three times with PBS. The cells $\left(1 \times 10^{4}\right)$ were incubated in the plate for $1 \mathrm{~h}$ and non-adherent cells were removed by washing with PBS. Cell viability was assessed by MTT $(0.5 \mu \mathrm{g})$ as described above.

The invasive activity of cells was determined using transwell chambers. Matrigel $(20 \mu \mathrm{l})$ (BD Biosciences, Bedford, MA, USA) at $500 \mu \mathrm{g} / \mathrm{ml}$ in cold PBS was spread on the inner side of the filter. The cells in the logarithmic phase were harvested and suspended at $1 \times 10^{5} / \mathrm{ml}$ in DMEM. Then, $100 \mu \mathrm{l}$ of cell suspension was added to the upper compartment and incubated at $37^{\circ} \mathrm{C}$ for $24 \mathrm{~h}$. Filters were subsequently fixed with $10 \%$ neutral formaldehyde for $30 \mathrm{~min}$ and stained with crystal violet for $5 \mathrm{~min}$. The cells on the upper side of the filter were removed with a cotton swab and any cells that had migrated through the Matrigel to reach the reverse side were counted under a microscope. 

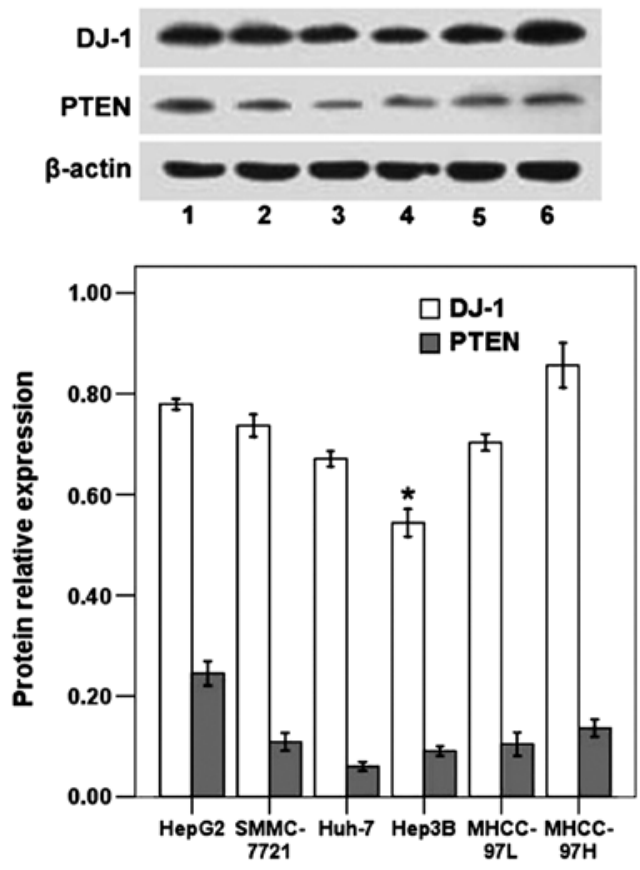

Figure 1. Expression levels of DJ-1 and PTEN in HCC cell lines. Extracts of HepG2, cell extracts from SMMC-7721, Huh-7, Hep3B, MHCC-97L and MHCC-97H were probed for DJ-1, PTEN and $\beta$-actin protein by immunoblotting. Quantities of DJ-1 and PTEN proteins were normalized to $\beta$-actin. Values present the means \pm standard deviation, to indicate a statistically significant result. ${ }^{*} \mathrm{P}<0.05$ was considered to indicate a statistically significant result. PTEN, phosphatase and tensin homolog.

Animal experiments. Six- to eight-week male BALB/c-nu mice were obtained from the Experimental Animal Center of the Huazhong University of Science and Technology (Wuhan, China). Animal experiments were conducted according to the guidelines of and approved by the Committee on Animals of the Huazhong University of Science and Technology. Animals were provided with food and water ad libitum and allowed one week adaptation. The mice were housed at $25 \pm 1^{\circ} \mathrm{C}$ under pathogen-free conditions with a 12-h light/dark cycle and $40-60 \%$ relative humidity. Single-cell suspensions (HepG2, RNAi-DJ-1 or RNAi-HK) were prepared and $5 \times 10^{6}$ cells in $0.2 \mathrm{ml}$ Hanks solution injected subcutaneously into mice. Tumor size was assessed every 4 days as $a b^{2} / 2$, where $a$ and $b$ are the major and minor diameter, respectively. Four weeks after the first measurement, the mice were sacrificed.

Statistical analysis. The results were expressed as means \pm standard deviation (SD). The SPSS 12.0 software (SPSS, Inc., Chicago, IL, USA) was used to perform the analysis. One-way ANOVA was used for comparisons among different groups. $\mathrm{P}<0.05$ was considered to indicate a statistically significant result.

\section{Results}

DJ-1 and PTEN expression in human HCC cell lines. The expression levels of DJ-1 and PTEN were assessed by immunoblot analysis in human HCC cell lines including HepG2, SMMC-7721, Huh-7, Hep3B, MHCC-97L and MHCC-97H. As shown in Fig. 1, DJ-1 expression varied significantly between cell lines. Significantly lower DJ-1 levels were
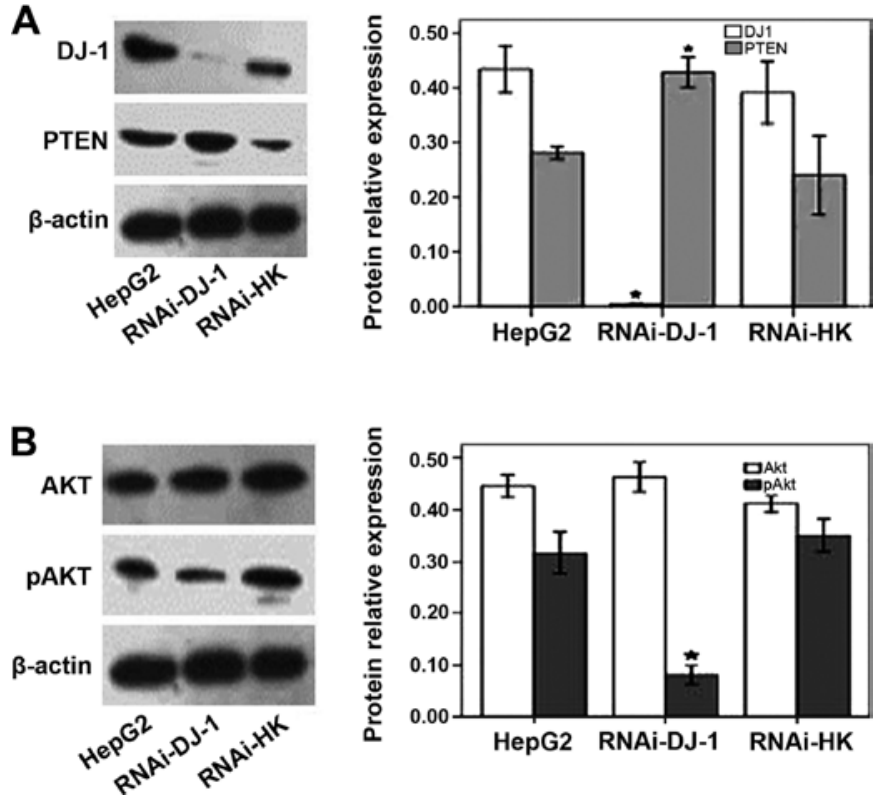

Figure 2. Effects of DJ-1 knockdown on PTEN and AKT in HepG2 cells. Cells were transfected with DJ-1 shRNA-expressing plasmids or control plasmid RNAi-HK for 8 weeks. The levels of the proteins in the control and shRNA cell lines were determined from whole cell extract using immunoblot analysis. (A) DJ-1 and PTEN protein expression levels. (B) Total and phoshorylated Akt (pAkt) levels. Quantities of proteins were normalized to $\beta$-actin. Values present means the \pm standard deviation, to indicate a statistically significant result. ${ }^{*} \mathrm{P}<0.05$ was considered to indicate a statistically significant result. PTEN, phosphatase and tensin homolog.

observed for Hep3B cells, which possess low metastatic potential, in comparison with the MHCC-97L cell line with low metastatic potential and the MHCC-97H cell line with high metastatic potential $(\mathrm{P}<0.05)$. The protein expression of DJ-1 in MHCC-97L cells was significantly lower than that in MHCC-97H cells $(\mathrm{P}<0.05$; Fig. 1). The results suggested that DJ-1 expression may correlate with the metastatic potential of HCC cell lines. Concerning PTEN expression, no significant differences were obtained when these cell lines were compared (Fig. 1). HepG2 cells were selected to examine the high level of DJ-1 expression in this cell line. This cell line has been widely used for the in vitro and in vivo studies of treatment of HCC and the mechanism involved.

shRNA knockdown of DJ-1 expression. The DNA segment encoding a shRNA for DJ-1 was inserted into a pGenesil-1 plasmid expression vector containing the human U6 promoter, between the BamHII and SalII restriction sites. The shRNA expression was directly driven by the cytomegalovirus promoter. The correct inserts in the RNAi plasmids shRNADJ-1 and negative control shRNA-HK were confirmed with DNA sequencing (data not shown). The plasmids were transfected into HepG 2 cells, with a transfection efficiency of $\sim 70 \%$ after $48 \mathrm{~h}$ (data not shown). After 8 weeks of G418 selection stable sub-clones expressing green fluorescent protein were isolated and expanded for subsequent assays (data not shown).

RT-PCR analysis confirmed that the DJ-1 shRNA recombinant plasmid inhibited DJ-1 mRNA expression in HepG2 cells (data not shown). DJ-1 protein expression levels were reduced by $98.78 \%$ after silencing (Fig. $2 \mathrm{~A}$ ). 
A
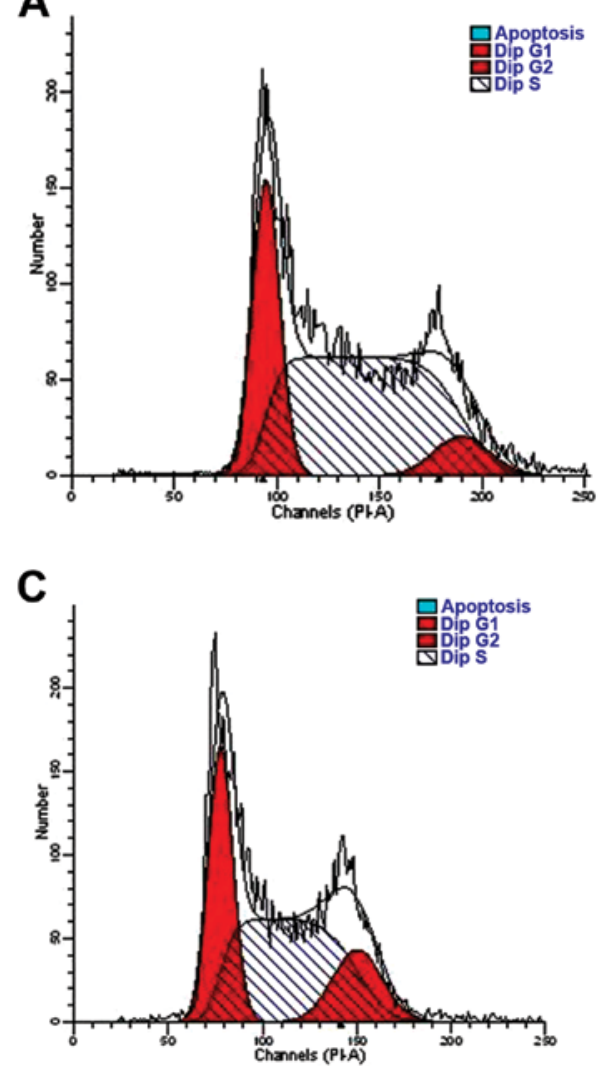

B
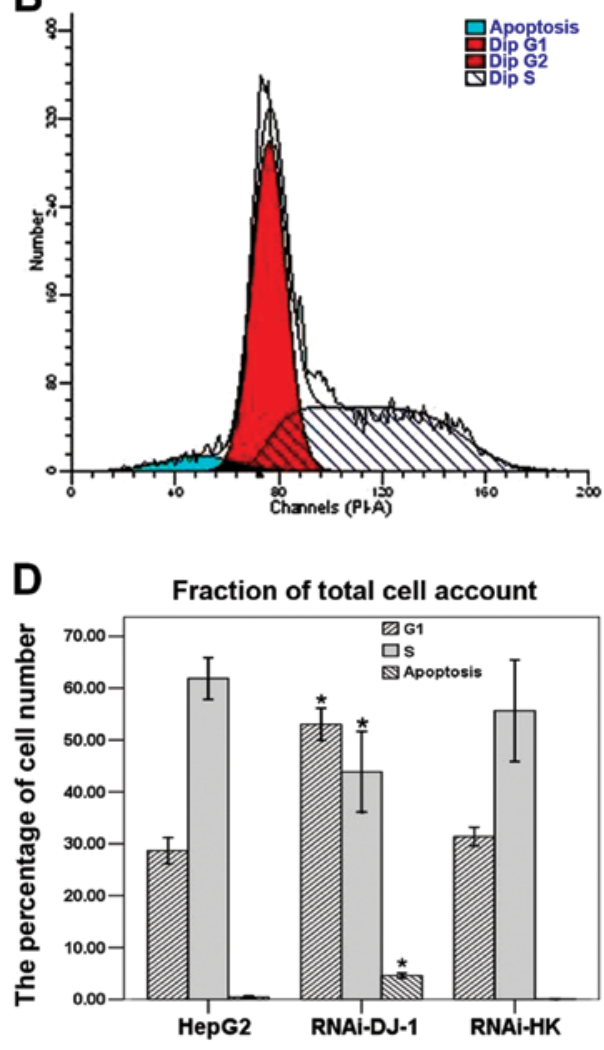

Figure 3. Impact of DJ-1 knockdown induced cell death. Cells were stained with propidium iodide staining and analyzed using flow cytometry. (A) The populations of cells in different cell cycle stages, as well as cells in apoptosis were estimated by measuring the area shown on the flow cytometry diagram. Compared to untreated cells, the (C) RNAi-HK-transfected cells showed similar cell population distribution. However, an increased number of cells with fewer DNA contents (indicating apoptosis) were observed in (B) RNAi-DJ-1 cells. The data were quantified as shown in (D). Results present the means \pm standard deviation of three independent experiments, to indicate a statistically significant result. ${ }^{*} \mathrm{P}<0.05$ was considered to indicate a statistically significant result. PTEN, phosphatase and tensin homolog.

DJ-1 shRNA inhibited Akt phosphorylation in HepG2 cells. In cells treated with RNAi-DJ-1, PTEN protein levels increased, compared to the untreated cells or cells transfected with the control plasmid RNAi-HK (P<0.05; Fig. 2A). Akt phosphorylation (pAkt) levels were significantly reduced in the RNAi-DJ-1-treated cells compared to the control cells ( $\mathrm{p}<0.05$; Fig. 2B). By contrast, total Akt levels were not significantly altered by RNAi-DJ-1 ( $P>0.05$; Fig. 2B). These data indicated that the downregulation of DJ-1 resulted in an increase of PTEN protein levels as well as the inhibition of Akt phosphorylation.

DJ-1 shRNA suppressed the proliferation, adhesion and invasion of HepG 2 cells. PI-stained cells were analyzed using flow cytometry. In RNAi-DJ-1-transfected cells, we found a significant decrease in cell populations with diploid DNA as well as an accumulation of apoptotic cells ( $\mathrm{P}<0.05$; Fig. 3$)$. These findings suggested that DJ-1 knockdown arrested the cell cycle and induced apoptosis.

Using MTT assay (Fig. 4A), we detected that the proliferation of RNAi-DJ-1-transfected cells was significantly reduced in comparison with untreated cells and cells transfected with the control plasmid ( $\mathrm{P}>0.05$; Fig. 4A).

Cell adhesion and invasion rates were also significantly reduced in DJ-1 knockdown cells: binding to fibronectin and laminin was reduced by 56.07 and $60.73 \%$ in RNAi-DJ-1- transfected cells, respectively $(\mathrm{P}<0.05$; Fig. 4B). Compared to untreated cells, there were $53.34 \%$ fewer RNAi-DJ-1 cells that migrated through a transwell filter after $24 \mathrm{~h}(\mathrm{P}<0.05$; Fig. 4C).

RNAi-DJ-1 suppression of xenograft tumor formation in nude mice. Thirty nude mice received subcutaneous injections of normal HepG2 cells with or without transfected shRNA plasmid. As shown in Fig. 5, the xenograft of untreated HepG2 induced tumor growth in transplanted animals in a time-dependent manner. The RNAi-HK-transfected cells, when transplanted, induced similar level of tumor growth in SD mice. However, tumor growth in the RNAi-DJ-1 group was significantly reduced as compared to that of normal HepG2 cells and cells transfected with RNAi-HK $(\mathrm{P}<0.05)$. These data indicated that inhibition of DJ-1 by shRNA inhibited the in vivo growth of HepG2 cells in the xenograft transplant.

\section{Discussion}

Results of the present study have shown that DJ-1 was expressed in a variety of human HCC cell lines including HepG2, SMMC-7721, Huh-7, Hep3B, MHCC-97L and MHCC-97H, and that DJ-1 expression correlated positively with the metastatic potential of HCC cell lines (22). However, Zhang et al recently described DJ-1 downregulation in the early stages of HBV-infected HCC, suggesting an abnormal 

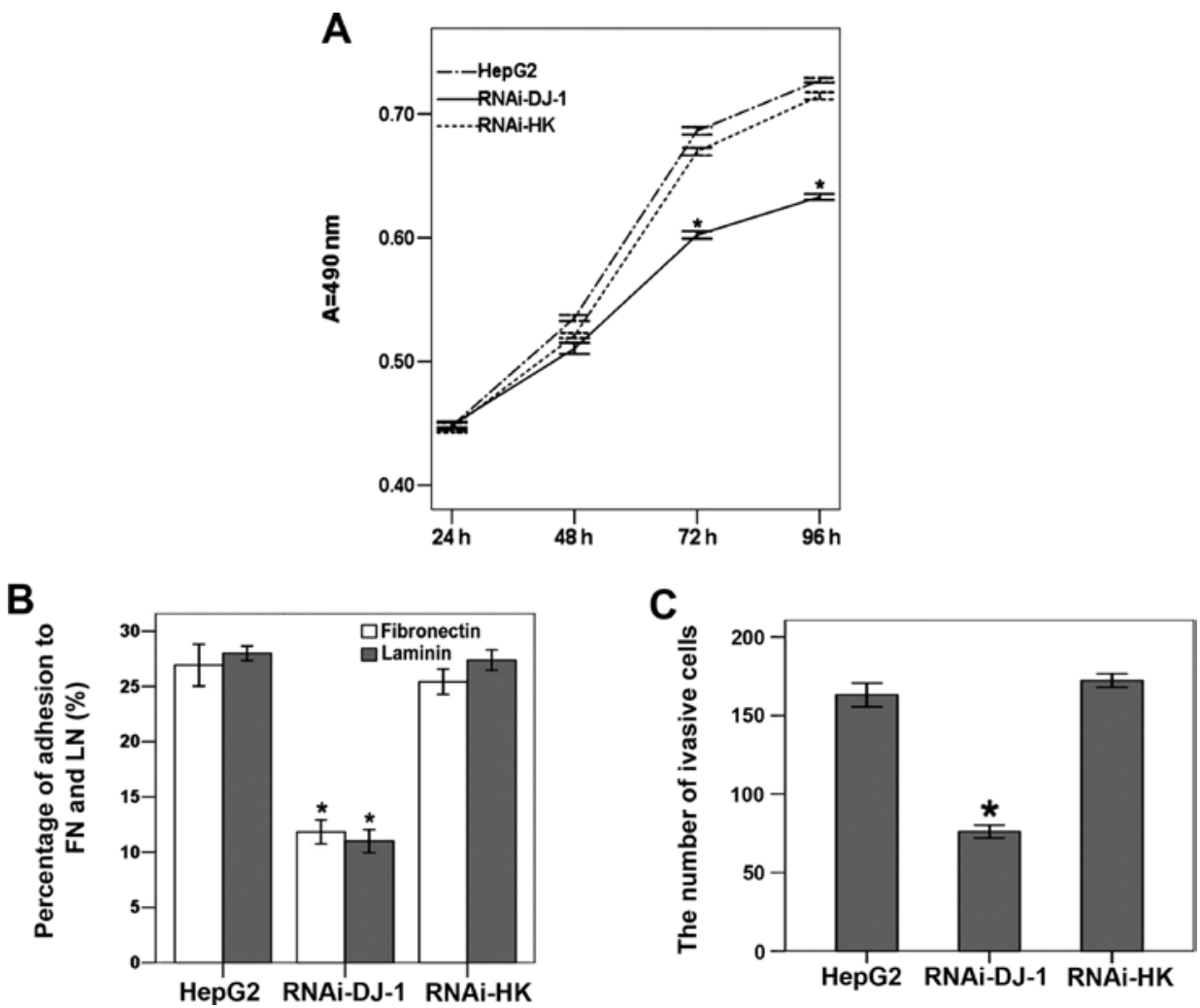

Figure 4. Impact of DJ-1 shRNA on HepG2 cell growth, adhesion and invasion. The abilities of cell growth (MTT assay), adhesion and invasion were tested in RNAi-DJ-1 cell lines as well as in control cells as described in Materials and methods. (A) Growth curves of transfectant HepG2 cells. (B) Transfectant HepG2 adhesion to fibronectin and laminin. (C) Invasion of transfectant HepG2 through transwell filters. Data represent means \pm standard deviation obtained from three independent experiments, to indicate a statistically significant result. ${ }^{*} \mathrm{P}<0.05$ was considered to indicate a statistically significant result. PTEN, phosphatase and tensin homolog.
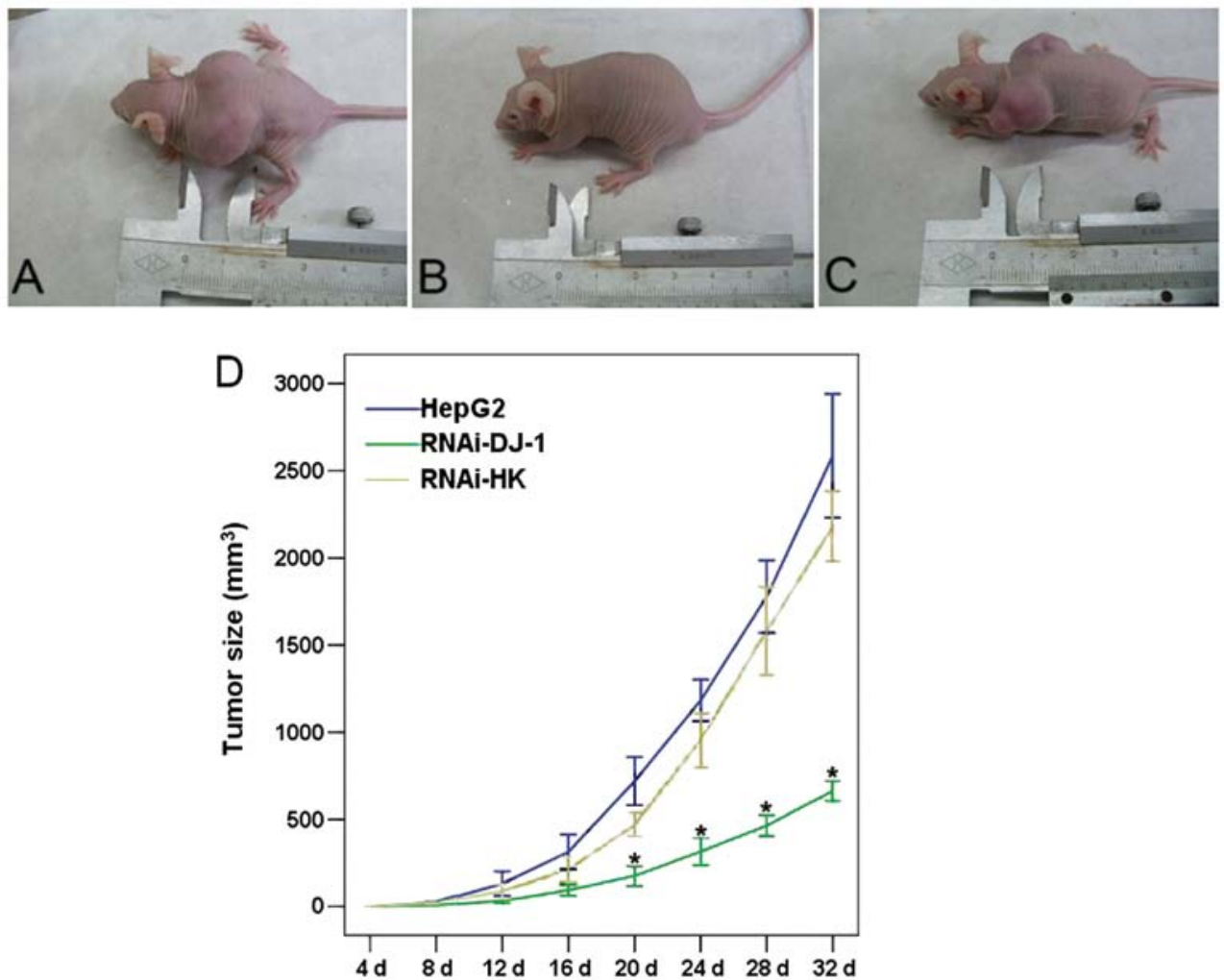

Figure 5. Effects of DJ-1 shRNA on tumor formation in vivo. HepG2 and HepG2 transfected with RNAi-DJ-1 and RNAi-HK were inoculated subcutaneously into nude mice. Typical images of tumor formation in nude mice of the (A) HepG2, (B) RNAi-DJ-1, (C) RNAi-HK groups. (D) Tumor growth was monitored and tumor volumes calculated. Results present the means \pm standard deviation obtained from three independent experiments, to indicate a statistically significant result. ${ }^{*} \mathrm{P}<0.05$ was considered to indicate a statistically significant result. PTEN, phosphatase and tensin homolog. 
pathophysiological state of the mitochondria (17). Authors of that study suggested that mitochondrial disease occurs before tumorigenesis in HBV-infected HCC (17). The conflicting findings on DJ-1 levels may reflect the differences between tumorigenesis in HBV-infected primary cells and HCC cell lines (17). Nevertheless, the observations strongly suggest that DJ-1 is involved in HCC, although its precise role in tumorigenesis remains to be determined.

The effects of DJ-1 on cell proliferation and apoptosis have been reported in other studies. For examples, cells harboring DJ-1 are more resistant to UV-induced apoptosis than DJ-1 knockdown cells (9). Overexpression of DJ-1 in a prostatic benign hyperplasia cell line (BPH-1) or prostate carcinoma cells (PC-3) results in resistance to the apoptosis normally induced by cytotoxic agents (10). By contrast, knockdown of DJ-1 enhances apoptosis of prostate carcinoma cells (PC-3) when treated with the same toxic reagents $(11,12)$ as well as TNF-related apoptosis-inducing ligand/Apo-2L (TRAIL)-mediated apoptosis (13). Overexpression of DJ-1 blocks neuronal apoptosis by inhibiting the transcriptional silencing activity of the pyrimidine tract-binding proteinassociated splicing factor, or by preventing the translocation of Daxx from the nucleus to the cytoplasm, where Daxx activates the apoptotic signal-regulating kinase-1 death pathway (10). In the present study, we employed RNAi technology to construct a shRNA plasmid to silence DJ-1 mRNA and demonstrated DJ-1 expression was markedly reduced in HepG2 cells after knockdown. Notably, DJ-1 silencing resulted in decreased proliferation, adhesion and invasion of HepG2 cells in vitro and inhibited tumor formation in nude mice. These results are consistent with a previous study $(11,12)$ suggesting DJ-1 in HepG2 cells plays a similar role in inhibiting apoptosis and promoting cell proliferation, adhesion and invasion.

DJ-1 was identified as a suppressor of the PTEN function through a genetic screening in Drosophila for gain-of-function mutants (16). PTEN negatively regulates the Akt signaling pathway through the dephosphorylation of PIP3 $(23,24)$. Activation of Akt is involved in the regulation of cell proliferation, apoptosis and migration (25).

We found that DJ-1 knockdown in HepG2 cells resulted in the upregulation of PTEN and decreased Akt phosphorylation. These results suggest that the PI3P/Akt is the potential signaling pathway in which DJ-1 functions.

In summary, the downregulation of DJ-1 increased PTEN expression, and decreased phosphorylation of Akt in HepG2 cells. In addition, DJ-1 knockdown resulted in decreased proliferation, adhesion and invasion of HepG2 cells in vitro, and inhibited the growth of HepG2-induced tumor in vivo. These findings suggest that DJ-1 plays a crucial role in the oncogenesis of HCC, thereby providing a potential target for drug development for HCC.

\section{Acknowledgements}

The present study was supported by the Ph.D. Programs Foundation of the Ministry of Education of China (project no. 20130142120043).

\section{References}

1. Llovet JM, Burroughs A and Bruix J: Hepatocellular carcinoma. Lancet 362: 1907-1917, 2003.

2. Giannelli G and Antonaci S: Novel concepts in hepatocellular carcinoma: from molecular research to clinical practice. J Clin Gastroenterol 40: 842-846, 2006.

3. Sherman M: Hepatocellular carcinoma: epidemiology, surveillance, and diagnosis. Semin Liver Dis 30: 3-16, 2010.

4. Rahbari NN, Mehrabi A, Mollberg NM, et al: Hepatocellular carcinoma: current management and perspectives for the future. Ann Surg 253: 453-469, 2011.

5. Adachi E, Maehara S, Tsujita E, et al: Clinicopathologic risk factors for recurrence after a curative hepatic resection for hepatocellular carcinoma. Surgery 131 (Suppl 1): S148-S152, 2002.

6. Diamantis A, Magiorkinis E, Sakorafas GH and Androutsos G: A brief history of apoptosis: from ancient to modern times. Onkologie 31: 702-706, 2008.

7. Fabregat I: Dysregulation of apoptosis in hepatocellular carcinoma cells. World J Gastroenterol 15: 513-520, 2009.

8. Schulze-Bergkamen H and Krammer PH: Apoptosis in cancerimplications for therapy. Semin Oncol 31: 90-119, 2004.

9. Mo JS, Kim MY, Ann EJ, Hong JA and Park HS: DJ-1 modulates UV-induced oxidative stress signaling through the suppression of MEKK1 and cell death. Cell Death Differ 15: 1030-1041, 2008.

10. Junn E, Taniguchi H, Jeong BS, Zhao X, Ichijo H and Mouradian MM: Interaction of DJ-1 with Daxx inhibits apoptosis signal-regulating kinase 1 activity and cell death. Proc Natl Acad Sci USA 102: 9691-9696, 2005.

11. Yuen HF, Chan YP, Law S, et al: DJ-1 could predict worse prognosis in esophageal squamous cell carcinoma. Cancer Epidemiol Biomarkers Prev 17: 3593-3602, 2008.

12. Davidson B, Hadar R, Schlossberg A, et al: Expression and clinical role of DJ-1, a negative regulator of PTEN, in ovarian carcinoma. Hum Pathol 39: 87-95, 2008.

13. Zhang HY, Wang HQ, Liu HM, Guan Y and Du ZX: Regulation of tumor necrosis factor-related apoptosis-inducing ligandinduced apoptosis by DJ-1 in thyroid cancer cells. Endocr Relat Cancer 15: 535-544, 2008.

14. Hod Y: Differential control of apoptosis by DJ-1 in prostate benign and cancer cells. J Cell Biochem 92: 1221-1233, 2004.

15. MacKeigan JP, Clements CM, Lich JD, Pope RM, Hod Y and Ting JP: Proteomic profiling drug-induced apoptosis in non-small cell lung carcinoma: identification of RS/DJ-1 and RhoGDI $\alpha$. Cancer Res 63: 6928-6934, 2003.

16. Kim RH, Peters M, Jang Y, et al: DJ-1, a novel regulator of the tumor suppressor PTEN. Cancer Cell 7: 263-273, 2005.

17. Zhang D, Lim SG and Koay ES: Proteomic identification of down-regulation of oncoprotein DJ-1 and proteasome activator subunit 1 in hepatitis B virus-infected well-differentiated hepatocellular carcinoma. Int J Oncol 31: 577-584, 2007.

18. Das R, Mahabeleshwar GH and Kundu GC: Osteopontin stimulates cell motility and nuclear factor $\kappa \mathrm{B}$-mediated secretion of urokinase type plasminogen activator through phosphatidylinositol 3-kinase/Akt signaling pathways in breast cancer cells. J Biol Chem 278: 28593-28606, 2003.

19. McManus MT and Sharp PA: Gene silencing in mammals by small interfering RNAs. Nat Rev Genet 3: 737-747, 2002.

20. Wang SL, Yao HH and Qin ZH: Strategies for short hairpin RNA delivery in cancer gene therapy. Expert Opin Biol Ther 9: 1357-1368, 2009.

21. Romashkova JA and Makarov SS: NF- $\kappa$ B is a target of AKT in anti-apoptotic PDGF signalling. Nature 401: 86-90, 1999.

22. Li Y, Tang ZY, Ye SL, et al: Establishment of cell clones with different metastatic potential from the metastatic hepatocellular carcinoma cell line MHCC97. World J Gastroenterol 7: 630-636, 2001.

23. Song MS, Salmena L and Pandolfi PP: The functions and regulation of the PTEN tumour suppressor. Nat Rev Mol Cell Biol 13: 283-296, 2012.

24. Sun H, Lesche R, Li DM, et al: PTEN modulates cell cycle progression and cell survival by regulating phosphatidylinositol 3,4,5,-trisphosphate and Akt/protein kinase B signaling pathway. Proc Natl Acad Sci USA 96: 6199-6204, 1999.

25. Mitsiades CS, Mitsiades N and Koutsilieris M: The Akt pathway: molecular targets for anti-cancer drug development. Curr Cancer Drug Targets 4: 235-256, 2004. 